\title{
BRAIN METABOLITES IN PRETERM GREY AND WHITE MATTER AND ALTERATION
} WITH INJURY

\author{
D. Card ${ }^{1}$, A.M. Moore ${ }^{2}$, J.G. Sled ${ }^{3}$, H.E. Whyte ${ }^{2}$, M.J. Taylor ${ }^{1}$ \\ ${ }^{1}$ Diagnostic Imaging, The Hospital for Sick Children, ${ }^{2}$ Neonatology, The Hospital for Sick Children, \\ University of Toronto, ${ }^{3}$ Physics and Experimental Medicine, The Hospital for Sick Children, Toronto, ON, \\ Canada
}

Background: Magnetic resonance spectroscopic imaging provides a metabolic snapshot of the brain. The objective of this study was to determine how ratios of the metabolites N-Acetylaspartate (NAA), Choline (Cho) and Creatine (Cr) vary with tissue type and injury in the preterm brain.

Methods: 43 preterm infants $(29 \pm 1.7$ wks GA), were scanned within two weeks of birth on a $1.5 \mathrm{~T}$ MRI (GE). Spectroscopy data were collected and analyzed using LCModel. In-house software was used to segment the brain into white matter (WM), grey matter (GM). Spectroscopy data was regressed against the relative proportion of GM in each voxel to estimate metabolite ratios in pure GM and pure WM for each subject and analysed according to age at scan and presence or absence of structural brain injury.

Results: 21 neonates had normal structural scans; germinal matrix hemorrhage was identified in 12 , cerebellar lesions in 2 and white matter injury in 15. Mean NAA/Cho values were $1.20 \pm 0.21(\mathrm{GM})$ and 0.97 \pm 0.25 (WM). Mean NAA/Cr values were $1.08 \pm 0.14(\mathrm{GM})$ and $0.77 \pm 0.26(\mathrm{WM})$. Both ratios increased with age and were significantly higher in GM, $<<0.05$ than WM. The NAA/Cho ratio in GM was significantly lower in the injured compared to the normal group, $\mathrm{p}<0.05$.

Conclusions: NAA/Cho and NAA/Cr increased with age and were significantly higher in GM than WM, although with brain lesions, NAA/Cho was significantly lower in GM. Combined with outcome data, these findings should lead to better understanding of preterm brain development and injury. 